\begin{tabular}{|c|l|}
\hline Title & Evolution of the maturation rate coll lapses competitive coexistence \\
\hline Author(s) & Mougi, A kihiko; Nishimura, Kinya \\
\hline Citation & $\begin{array}{l}\text { Journal of Theoretical Biology, 241(3), 467-476 } \\
\text { https://loi.org/10.1016/.jtbi.2005.12.017 }\end{array}$ \\
\hline Issue Date & $2006-08$-07 \\
\hline Doc URL & http://hdl.handle.net/2115/14664 \\
\hline Type & article (author version) \\
\hline File Information & JTB2006-241-3.pdf \\
\hline
\end{tabular}

Instructions for use 


\section{Evolution of the maturation rate collapses competitive coexistence}

Akihiko Mougi $†$ and Kinya Nishimura $\ddagger$

Graduate School of Fisheries Sciences

Hokkaido University

Hakodate 041-8611, Japan

10

To whom correspondence should be addressed

†mougi@ fish.hokudai.ac.jp

łkinya@fish.hokudai.ac.jp

15 


\section{Abstract}

Most theoretical studies on character displacement and the coexistence of competing species have focused attention on the evolution of competitive traits driven by interspecific competition. We investigated the evolution of the maturation rate which is not directly related to competition and trades off with the birth rate and how it influences competitive outcomes. Evolution may result in the superior competitor becoming extinct if, initially, the inferior competitor has a lower, and the superior one a higher, maturation rate at the coexistence equilibrium. This counterintuitive result is explained by an explosive increase in the adult population of the inferior competitor as a

10 result of the more rapid evolution of its maturation rate, which is caused by differences in the intensity and direction of selection on the maturation rates of the two species and in their adult densities, which are related to differences in their life histories. Thus, a life-history trait trade-off with a competitive trait may cause a competitive ecological coexistence to collapse.

15

Keywords: competition, coexistence, life-history traits, maturation rate, adaptive dynamics 


\section{Introduction}

Classical ecological theory predicts that two competing species both sharing a limited resource cannot coexist in the same locality (Lotka 1925; Gause 1934; MacArthur 1972). This theory unambiguously predicts that the superior competitor excludes the

5 inferior competitor. In reality, however, this expectation is often belied (Paine 1966;

Grover 1997; Levines \& Rees 2002).

Empirical and theoretical studies suggest that a compensatory balance between the performance of several life-history traits and competitive ability may exist among competing species. This balance is the most common mechanism used to explain the

10 discrepancy between the observed coexistence of competing species and the predictions of classical competition theory (Hutchinson 1957; Tilman 1982; Chase \& Leibold 2003). Even though the inferior competitor suffers a competitive disadvantage, that disadvantage can be compensated for by some advantage conferred by its other life-history traits. For instance, if a trade-off exists between colonization rate and competitive ability (Hutchinson 1951; Levins \& Culver 1971; Nee \& May 1992; Levine \& Rees 2002), or if the superior competitor has a lower tolerance for a predator or the abiotic environment than the inferior competitor (Paine 1966; Armstrong 1979; Tilman \& Pacala 1993), coexistence may be possible. 
However, we do not know whether competitive coexistence on an ecological time scale made by a compensatory balance of life-history traits and competitive abilities between the competing species is maintained under an evolutionary process of life-history traits. Life-history traits can be an object of natural selection (Sterns 1977).

5 Nakajima and Kurihara (1994) and Yoshida et al. (2003), on the basis of empirical studies, suggested that life-history traits evolve as species interact and that their evolution influences in turn the dynamics of that interaction. Although classical theory attempts to link character evolution with ecological competitive interaction (MacArthur \& Levins 1967; May \& MacArthur 1972; Roughgarden 1972; Slatkin 1980; Case 1981;

10 Taper \& Case 1985, 1992a, 1992b; Abrams \& Chen 2002b), no studies that we are aware of have focused on the problem as to whether the evolution of life-history traits maintains or promotes competitive coexistence accomplished by a compensatory balance between competitive abilities and life-history traits.

Theoretical studies on character displacement have usually attempted to understand competitive interaction through the evolution of competitive traits (MacArthur \& Levins 1967; May \& MacArthur 1972; Roughgarden 1972; Slatkin 1980; Case 1981; Taper \& Case 1985, 1992a, 1992b; Abrams \& Chen 2002b). Interspecific competitive relations are parameterized as competition coefficients by the Lotka-Volterra 
competition model, and niche theory relates these competition coefficients to biological reality; that is, among competing species, resource use overlaps on resource niche axes, and the characters related to use of the resource niche also overlap (MacArthur 1958; MacArthur \& Levins 1967; Vandermeer 1972; Pianka 1976; Hutchinson 1978). beak size are implicitly included in the competition coefficients of competition models of the Lotka-Volterra type. Theoretical studies on character displacement predict that interspecific competition triggers evolution of the traits of one or both of the competing species when any of those traits are directly related to a niche axis. Such character shifts not infrequently moderate interspecific competitive interaction (MacArthur \& Levins 1967; May \& MacArthur 1972; Roughgarden 1972; Slatkin 1980; Case 1981; Taper \& Case 1985, 1992a, 1992b; Abrams \& Chen 2002b). However, life-history traits not directly related to the competitive interaction might also evolve through interspecific competition (Abrams 2000; Abrams \& Chen 2002a).

15 Explicit specification of some life-history traits embedded in the parameter, that is, the competition coefficient, in a simple competition model of the Lotka-Volterra type can shed light on the mechanism of coexistence of competing species that would lead to balance between the functions of the niche-axis traits (direct effect either by interference 
or exploitative competition) and the functions of the life-history traits. Previously, we developed a stage-structured two-species competition model and analyzed the conditions required for stable coexistence of the competing species (Mougi \& Nishimura, 2005). We considered two species, each with distinct juvenile and adult 5 stages, in which intra- and interspecific competition occurs only within each ontogenetic life stage and not between stages. That is, each species has a complex life cycle and undergoes an ontogenetic niche shift (Werner \& Gilliam 1984). Our model, in which maturation rate, intrinsic birth rate, and potential death rate are life-history parameters explicitly specified to be other than the niche-axis traits embedded in the competition coefficients, shows that stable coexistence of competing species depends on life-history traits.

In this paper, we refer to that model and show, first, that coexistence of competing species is accomplished by a compensatory balance between competitive abilities and interrelated life-history traits. Second, we show that interspecific competition exerts selection pressure on a life-history trait. We focus on the maturation rate as the life-history trait targeted by selection, because there is often a trade-off between this trait, which is one of the most important life-history traits, and the birth rate (Sterns 1992; Roff 2000). Third, we show that the evolution of this life-history trait in the 
competing species does not necessarily maintain their coexistence.

\section{Model}

As a first step, we develop an expanded version of our stage-structured competition model (Mougi \& Nishimura, 2005), in which the interrelated life-history traits, maturation rate and birth rate, and competition coefficients reflecting niche-axis traits of both species define phenomena on an ecological timescale. We survey the conditions of stable coexistence for different combinations of competition coefficients and maturation rates for each species. Second, we examine evolution of the maturation rate as driven by the competitive relation, investigating in particular how the maturation rates of the

10 competing species evolve. Third, we evaluate the ecological consequence, that is, coexistence or exclusion of one of the two species, associated with the trait's evolution.

\section{2-1. Ecological dynamics}

Consider two competing species, one a superior and the other an inferior competitor, with a life cycle consisting of juvenile and adult stages (Wilbur 1980). Newborn

15 individuals first spend time in the juvenile stage, and, if they survive and mature, reach the adult stage, during which they reproduce. Reproduction is diminished by intra- and interspecific competition among adults, and we assume that there is ontogenetic niche separation between the adult and juvenile stages (Werner \& Gilliam 1984). 
Dynamics of a stage-structured system of two competing species reflecting these scenarios are described by the following equations:

$$
\begin{aligned}
& \dot{X}=\gamma_{x} x-c_{x} X, \\
& \dot{x}=b_{x}(X, Y) X-\gamma_{x} x-\phi_{x}(x, y) x, \\
& \dot{Y}=\gamma_{y} y-c_{y} Y, \\
& \dot{y}=b_{y}(X, Y) Y-\gamma_{y} y-\phi_{y}(x, y) y .
\end{aligned}
$$

where $c_{x}$ and $c_{y}$ are per capita adult mortality rates, and $\gamma_{x}$ and. $\gamma_{y}$ are per capita maturation rates of species- $X$ and species- $Y$, respectively. $X$ and $Y$ are the adult densities of the two species, $x$ and $y$ are the juvenile densities; $\alpha$ and $\beta$ are the interspecific competition coefficients for adults; and $\alpha^{\prime}$ and $\beta^{\prime}$ are the interspecific competition coefficients for juveniles. $b_{x}(X, Y)$ and $b_{y}(X, Y)$ are the per capita birth rates of species- $X$ and species- $Y$, respectively. Each is a linear decreasing function with respect to the density of conspecific and heterospecific individuals through which competition among adults is realized, that is, $b_{x}(X, Y)=b\left(\gamma_{x}\right)(1-X-\alpha Y), b_{y}(X, Y)=b\left(\gamma_{y}\right)(1-Y-\beta X) . b\left(\gamma_{x}\right)$ and $b\left(\gamma_{y}\right)$ are the intrinsic birth rates of species- $X$ and species- $Y$, respectively, each of which is a function of the maturation rate $\gamma$ (see the next paragraphs). $\phi_{x}(x, y)$ and $\phi_{y}(x, y)$ are per capita juvenile mortality rates of species- $X$ and species- $Y$, respectively, and each of these is also a linear increasing function with respect to the density of conspecific and 
heterospecific individuals through which competition among juveniles is realized; that is, $\phi_{x}(x, y)=\phi+x+\alpha^{\prime} y, \phi_{y}(x, y)=\phi+y+\beta^{\prime} x$.

The life-history trait on which we focus, the maturation rate, determines the duration of development before maturation, which is reflected in the birth rate. We assume that 5 individuals with a low maturation rate (i.e., those who remain longer in the juvenile stage on average and thus mature at an older age) produce many offspring (because of their large body size). In contrast, individuals with a high maturation rate (i.e., those who remain for a shorter time on average in the juvenile stage, and thus mature at a younger age) produce few offspring (because of their small body size). This trade-off is a common feature of various taxa (e.g., Salmo gairdneri) (Roff 2000).

$b\left(\gamma_{x}\right)$ and $b\left(\gamma_{y}\right)$ are the intrinsic birth rates of species- $X$ and species- $Y$, respectively, as a function of the maturation rate $\gamma$. For mathematical simplicity, we adopt the linear trade-off function $b\left(\gamma_{i}\right)=b_{i}-\lambda_{i} \gamma_{i}$, where $b_{i}$ is the baseline intrinsic birth rate and $\lambda_{i}$ is the intensity of the trade-off between the maturation rate and the intrinsic birth rate, to describe this trade-off phenomenon. Relaxation of this restrictive simplification does not influence our main results (which remain the same even if we use another trade-off function such as $b\left(\gamma_{i}\right)=\left(b_{i}-\lambda_{i} \gamma_{i}\right)^{p}$ for $\left.0<p\right)$.

\section{2-1-1. Ecological equilibrium}


First, we analyze the ecological outcomes without allowing the life-history traits to evolve. We concentrate on a particular competitive relation, dominance competition, in which species- $X$ enjoys dominance $\left(\alpha^{\prime}<1, \alpha<1, \beta^{\prime}>1\right.$ and $\left.\beta>1\right)$. Species- $X$ is, hence, the superior competitor, and species- $Y$ is the inferior competitor. For simplicity, we assume that all parameters except for the competition coefficients and maturation rates are identical between the species, and that the competitive relation does not change ontogenetically (that is, $\alpha^{\prime}=\alpha$ and $\beta^{\prime}=\beta$ ).

We analyzed the conditions required for the existence of a positive nontrivial equilibrium (with all elements of the equilibrium, $X^{*}, x^{*}, Y^{*}$, and $y^{*}$, positive) and for its stability. Stable coexistence does not occur if the maturation rates of both species are identical (i.e., along the $45^{\circ}$ diagonal in Fig. 1(a) or (b)). However, coexistence is possible if the superior competitor has a lower maturation rate (implying a lower turnover rate or longer generation time, even though a higher fecundity) (region $I a$ in Fig. 1(a) and (b)) or a higher maturation rate (implying lower fecundity or a lower intrinsic birth rate, even though a faster turnover rate) (region $I b$ in Fig. 1(a) and (b)) compared with the inferior competitor, even though coexistence is restricted to narrow ranges of the set of possible maturation rates when the competitive abilities of the inferior and superior species are similar (see Fig. 1(b)). These results suggest that a 
balance between competitive ability and another life-history trait (maturation rate) makes the coexistence of the competing species possible.

\section{2-2. Evolutionary dynamics}

Here, we demonstrate that competitive interaction causes one life-history parameter, the

5 maturation rate, to evolve, although it is not a niche-axis trait directly related to the competitive interaction process.

We use an adaptive dynamics framework to determine which maturation strategies are favored by natural selection. "Adaptive dynamics" is a theoretical framework for bridging the gap between micro- and macroevolution (Dieckmann, 1997, Geritz, 1998).

10 It is based on two main simplifying assumptions: a separation between the population dynamic and mutational timescales, and clonal genetics. Evolution proceeds by the continual replacement of members of the resident population by novel mutants. The latter originate by chance, but their evolutionary fate depends on their fitness, that is, their capacity to increase their numbers.

15 We investigate how the coexistence maintained by the ecological dynamics (eqs. (1a)-(1d)) is affected by the evolution of the maturation rate.

\section{2-2-1. Fitness of a mutant}

We define the fitness of a mutant in each resident population as the expected lifetime 
reproduction rate per born individual. Consider a population of species $i(i=X$ or $Y)$ in which all individuals have a maturation strategy, $\gamma_{i}$. The fitness of a rare mutant with maturation strategy $\hat{\gamma}_{i}$, which is infinitesimally close to the value of $\gamma_{i}$, is denoted $W_{i}\left(\hat{\gamma}_{i}, \gamma_{i}\right)$

A newborn mutant individual with maturation strategy $\hat{\gamma}_{i}$ survives and definitely matures with probability $M_{i}=\hat{\gamma}_{i} /\left(\hat{\gamma}_{i}+\phi_{i}\left(x^{*}, y^{*}\right)\right)$, and the survivors are in the juvenile stage for a period of time $L_{i}=1 / \hat{\gamma}_{i}$, and in the adult stage for a period of time $R_{i}=1 / c_{i}$, during which the instantaneous birth rate is $B_{i}=b\left(\hat{\gamma}_{i}\right) F_{i}\left(F_{x}=1-X^{*}-\alpha Y^{*}, F_{y}=1-Y^{*}\right.$ $\left.-\beta X^{*}\right)$ at ecological equilibrium. The expected lifetime reproduction rate per born individual is calculated by dividing the expected lifetime reproduction, $M_{i} R_{i} B_{i}$, by the expected lifetime, $L_{i}+R_{i}$. Thus, the fitness of a mutant of either species is

$$
W_{i}\left(\hat{\gamma}_{i}, \gamma_{i}\right)=\frac{M_{i} R_{i} B_{i}}{L_{i}+R_{i}}
$$

Whether the mutant, through its mutant strategy, can invade the resident population is evaluated by determining the local fitness gradient,

$\left.15 \quad \frac{\partial W_{i}\left(\hat{\gamma}_{i}, \gamma_{i}\right)}{\partial \hat{\gamma}_{i}}\right|_{\hat{\gamma}_{i}=\gamma_{i}}$.

If the gradient is positive, then mutants with a higher maturation rate than that of the resident population can invade that population, and, conversely, if it is negative, then those with a lower maturation rate than that of the resident population can invade that 
population.

\section{2-3. Extinction of the superior competitor}

We investigate how the life-history trait evolves in the competitive interaction, and how the trait evolution affects the coexistence of the two species. We assume that evolution

5 is strictly mutation-limited (i.e., a new mutant enters only when the former population has reached a stationary state). If we assume that ecological and evolutionary processes operate at different timescales, then the rates of change of traits are given by $u_{i} b_{i}\left(X^{*}\right.$, $\left.Y^{*}\right) N_{i}^{*}\left(\partial W_{i}\left(\hat{\gamma}_{i}, \gamma_{i}\right) / \partial \hat{\gamma}_{i}\right) \hat{\gamma}_{i}=\gamma_{i}$, where $\left(i=X\right.$ or $Y$, and $N_{i}^{*}=X^{*}$ or $\left.Y^{*}\right)($ Dieckmann $\&$ Law 1996). The parameter $u_{i}$ denotes the mutation rate, and $b_{i}\left(X^{*}, Y^{*}\right)$ implies the instantaneous birth rates of the resident populations of species- $X$ or species- $Y$.

First, we analyze the direction and intensity of selection acting on the trait by evaluating the magnitude and sign of the selection gradient, $\left(\partial W_{i}\left(\hat{\gamma}_{i}, \gamma_{i}\right) / \partial \hat{\gamma}_{i}\right)_{\hat{\gamma}_{i}}=\gamma_{i}$. Figure 2 shows the vector fields based on the magnitude and sign of the fitness gradient for the maturation rates of two species superimposed on the maturation rates plane under ecological equilibrium conditions. It is clear that the direction of the selection gradient vector necessarily trends toward extinction of the inferior competitor when the competition effect of the inferior competitor on the superior is very low (Fig. 2(a), $\alpha=$ 0.1 ). If the interspecific competition intensity offered by the inferior competitor is 
increased (Fig. 2(b1), $\alpha=0.9$ ), however, the direction of the selection gradient vector trends toward extinction of the superior competitor (see Fig. 2(b2)).

As shown in the figure, the maturation rate of the superior competitor decreases and that of the inferior competitor increases, and the selection intensity for the maturation rate of the inferior competitor is much stronger than for that of the superior competitor. The result does not change for different values of $\lambda$ and is hardly influenced by the form of the trade-off function. In the following, we focus on the unexpected trend that the direction of the fitness gradient leads to the extinction of the superior competitor, and investigate whether selection acting on this trait in each species really causes the extinction of the superior species via adaptive evolution.

\section{2-3-1. Mechanisms causing the direction of the fitness gradient trend toward the extinction of the superior competitor}

In order to understand the mechanisms causing the selection gradient vector to trend toward extinction of the superior competitor (see Fig. 2(b2)), we focus on two questions.

15 First, why is the direction of evolution of the trait different for the two species (the maturation rate of the inferior competitor becomes higher, and that of the superior competitor becomes lower). Second, why does the intensity of selection differ between the two species (i.e., stronger in the inferior competitor and weaker in the superior 
competitor)?

\section{2-3-1-1. Invasible traits}

Suppose that a trait set that satisfies the conditions for the coexistence of the two competing species exists (e.g., a point in region $I b$ of Fig. 2(b2)). Selection acts on the

5 traits of the two species in opposite directions (as indicated by the directions of the component vectors of the decomposed synthetic vector in region $I b)$. The selection pressures drive the maturation rate of the inferior competitor toward higher values and that of the superior competitor toward lower values.

To clarify the mechanisms generating this difference in direction, we investigated the conditions under which a mutant invades a resident population. We can determine whether a strategy of a higher or a lower maturation rate will allow the invasion by examining the sign, positive or negative, of the selection gradient for a given value of maturation rate of the resident population. The condition that a higher maturation rate strategy will enable a mutant to invade the resident population of species $i(i=X$ or $Y)$,

$$
-\frac{b^{\prime}\left(\gamma_{i}\right)}{b\left(\gamma_{i}\right)}<\frac{1}{\gamma_{i}} \cdot\left\{\frac{c_{i}}{c_{i}+\gamma_{i}}+\frac{\phi_{i}\left(x^{*}, y^{*}\right)}{\phi_{i}\left(x^{*}, y^{*}\right)+\gamma_{i}}\right\},
$$

where $b^{\prime}\left(\gamma_{i}\right)=\partial b\left(\gamma_{i}\right) / \partial \gamma_{i}=-\lambda_{i}<0$.

Notice that both sides of the inequality are positive. This condition implies that a 
mutant having a higher maturation rate than the maturation rate of the resident population, that is, $\gamma_{i}<\hat{\gamma}_{i}$, can invade that population when the maturation rate of the resident population is low. In contrast, a mutant having a lower maturation rate than that of the resident population, that is $\hat{\gamma}_{i}<\gamma_{i}$, can invade when the maturation rate of the resident population is high.

The region where the mutant and the resident population can coexist, $I b$ of Fig. 2(b1), is located where values of $\gamma_{x}$ are relatively high and those of $\gamma_{y}$ are relatively low. Therefore, in that region a mutant with a lower maturation rate can invade a resident population of species- $X$, and a mutant with a higher maturation rate can invade a resident population of species- $Y$. Furthermore, ineq. (4) is likely to hold when the value of $c_{i}$ or $\phi_{i}\left(x^{*}, y^{*}\right)$ is large. Therefore, when the expected reproductive period, $1 / c_{i}$, is short or the probability that a born individual survives and definitely matures, $M_{i}=\gamma_{i} /\left(\gamma_{i}+\right.$ $\left.\phi_{i}\left(x^{*}, y^{*}\right)\right)$, is low, a mutant with a higher maturation rate strategy can invade.

\section{2-3-1-2. Strong intensity of selection on the inferior competitor}

We then investigated the difference in the selection intensity on the maturation rate in the region of coexistence (component vector magnitudes in region $I b$ of Fig. 2(b1)). The maturation rate of the inferior competitor experiences stronger selection pressure than that of the superior competitor. We examined the mechanism underlying this pattern of 
difference in the selection pressures to which the two species are subject.

The ratio of adults to juveniles in each species at ecological equilibrium is $X^{*} / x^{*}=$ $\gamma_{x} / c_{x}$ and $Y^{*} / y^{*}=\gamma_{y} / c_{y}$ (see Mougi \& Nishimura 2005). In the region of coexistence on the $\gamma_{x}-\gamma_{y}$ plane (region $I b$ of Fig. 2(b1)), the relative density of juveniles to that of adults 5 of the inferior competitor is high, and an invading mutant matures earlier than the resident population. Thus, the relative speed with which the frequency of the invading mutant increases in the adult stage in the inferior competitor is high. Furthermore, the intrinsic birth rate of the inferior competitor is high owing to its low maturation rate, because of the trade-off relationship between maturation rate and intrinsic birth rate.

10 Thus, the adults, which are the source of the new population, are likely to be rapidly replaced by the mutants with the higher maturation rate strategy in the inferior competitor.

Conversely, in the region of coexistence, the relative density of adults to juveniles of the superior competitor is high, and the advantageous mutant matures more slowly than 15 the resident population. Thus, the relative frequency of the mutant in the adult stage is low. Furthermore, the intrinsic birth rate of the superior competitor is low owing to its high maturation rate, because of the aforementioned trade-off. Therefore, replacement of the resident population by mutants in the superior competitor species is relatively 
slow.

\section{2-3-2. Adaptive evolutionary dynamics}

Next we describe the evolutionary dynamics by numerical simulations. The trajectory of adaptive evolutionary dynamics is determined by two elements, that is, not only by 5 the selection gradient, $\left(\partial W_{i}\left(\hat{\gamma}_{i}, \gamma_{i}\right) / \partial \hat{\gamma}_{i}\right)_{\hat{\gamma}_{i}=\gamma_{i}}(i=X$ or $Y)$, but also by the instantaneous occurrence rates of a mutant, $u_{i} b_{i}\left(X^{*}, Y^{*}\right) N_{i}^{*}\left(i=X\right.$ or $Y$, and $N_{i}^{*}=X^{*}$ or $\left.Y^{*}\right)$ (Dieckmann \& Law 1996). We iteratively replace a trait of the resident population of either species by a mutant trait with probabilities proportional to the magnitude of $u_{i} b_{i}\left(X^{*}, Y^{*}\right) N_{i}^{*}\left(\partial W_{i}\left(\hat{\gamma}_{i}, \gamma_{i}\right) / \partial \hat{\gamma}_{i}\right)_{\hat{\gamma}_{i}=\gamma_{i}} / \sum_{j \subset X, Y} u_{j} b_{j}\left(X^{*}, Y^{*}\right) N_{j}^{*}\left(\partial W_{j}\left(\hat{\gamma}_{j}, \gamma_{j}\right) / \partial \hat{\gamma}_{j}\right)_{\hat{\gamma}_{j}=\gamma_{j}}$, given the situation that the mutant emerges, thus making the time dimension implicit.

Even though the direction of the selection gradient vector necessarily trends toward extinction of the superior competitor in the coexisting region of the parameter space (Fig. 2(b2)), the effect of the instantaneous occurrence rates of a mutant in the superior and inferior competitors may act in the same or a different direction as the effect of the selection gradient vector. Thus, we have a separatrix, above which the evolutionary trajectory leads to extinction of the superior species and below which the trajectory leads to extinction of the inferior species (Fig. 2(b3)).

Below the separatrix the superior species (species- $X$ ) has a higher instantaneous 
occurrence rate of a mutant than the inferior species (species- $Y$ ) because species- $X$ has a larger number of adults than species- $Y$. The effect of the difference in the instantaneous occurrence rate of the mutant acts in a different direction than the effect of the selection gradient, and outweighs it. The combined effect leads to extinction of the inferior $5 \quad$ species below the separatrix (Fig. 2(b3), tr-2).

Above the separatrix, in contrast, either the effect of the selection gradient outweighs the effect of the instantaneous occurrence rates of the mutant, or the effect of the instantaneous occurrence rates of the mutant acts in the same direction as the effect of the selection gradient; thus, the combined effect leads to extinction of the superior species (Fig. 2(b3), tr-2). In the evolutionary trajectory toward extinction of the superior species, the relative speed of evolution is initially faster in the superior competitor because of its relatively higher adult density; however, the evolutionary speed in the inferior competitor gradually increases as the adult density of the superior decreases and that of the inferior increases. Therefore the rapid evolution of (strong selection pressure for) the inferior compared with the superior competitor leads to the extinction of the superior competitor.

\section{2-3-3. Adaptive life-history change and ecological extinction}

Adaptive trait evolution leading to extinction of the superior species is a remarkable 
consequence. Even though evolutionary change (i.e., trait evolution) and ecological change (i.e., ecological equilibrium state) have a mutual cause-effect relationship, a rapid evolutionary change in either or both species can cause a change in ecological equilibrium.

5 The rapid evolution of the maturation rate of the inferior competitor in the course of adaptive evolution (Fig. 2(b3), tr-1) can cause the extinction of the superior competitor. Since the evolutionary change in the maturation rate of the inferior competitor species predominates, we survey the change in the total density of each of the two species as $\gamma_{y}$ changes across the region of coexistence (Fig. 2(b3), tr-1).

10 The evolution toward a higher maturation rate in the competitively inferior species has consequences with reciprocal effect on the viability of that species. An increase in the maturation rate shortens the juvenile period, $L\left(\gamma_{y}\right)=1 / \gamma_{y}$, and thus increases the potential probability that a juvenile survives to become an adult, $M\left(\gamma_{y}\right)=\gamma_{y} /\left(\gamma_{y}+\phi_{y}\right)$. However, the increase of the maturation rate also decreases the intrinsic birth rate, $b\left(\gamma_{y}\right)$ $15=b_{y}-\lambda_{y} \gamma_{y}$, because the shortening of the juvenile period leads to a smaller adult body size.

A rapid increase of the maturation rate, $\gamma_{y}$, enhances the viability of the inferior species because its positive effects (the increase of $\tilde{M}\left(\gamma_{y}\right)$ and the decrease of $L\left(\gamma_{y}\right)$ ) 
greatly outweigh its negative effect (decrease of $b\left(\gamma_{y}\right)$ ), thus increasing the density of the inferior competitor species. The increase in the population density of the inferior competitor driven by the evolution of its own maturation rate toward higher values decreases the density of the superior competitor as well (see Appendix A for the proof).

5 Thus, its rapid evolution makes the inferior competitor species more viable, sufficiently offsetting its intrinsic weak competitive ability. Ultimately, this overcompensation effect causes the extinction of the superior competitor species (see Fig. 3).

\section{2-4. Evolutionarily stable coexistence}

Even though a compensatory balance between competitive abilities and viabilities determined by the maturation rates allows coexistence of two competing species on an ecological timescale, evolution of the maturation rates driven by their competitive interaction collapses that coexistence. The conditions of coexistence of competing species are more restricted on an evolutionary timescale than those inferred from coexistence theory based on an ecological timescale. coefficients and the evolutionary parameter, i.e., maturation rates is the setting of our model. In the parameters setting, a singular point of the maturation rates attained by the evolutionary process where the fitness gradient is zero, $\left(\tilde{\gamma}_{x}, \tilde{\gamma}_{y}\right)$, satisfies $\tilde{\gamma}_{x}=\tilde{\gamma}_{y}$, 
and the two speice cannot coexist at the singular point (see Appendix B).

If we relax the assumption of symmetrical parameters other than competition coefficients and maturation rates, we can investigate combinations of competition coefficients and life-history parameters that make coexistence stable on an evolutionary

5 timescale. For example, if we assume that $c_{x}>c_{y}$, the evolution of the maturation rate does not collapse the ecological coexistence, provided that the inferior competitor's competitive ability is much weaker $(\alpha \ll 1)$ than that of the superior competitor (see Fig. 2(c)).

In addition, an evolutionary stability analysis of the singular point shows that it is evolutionarily stable (ESS) (see Appendix C). This means that coexistence is evolutionarily stable at that point.

\section{Discussion}

A direct trade-off between competitive abilities and other life-history traits is a common explanation for competitive coexistence in communities (Kneitel \& Chase 2004). In this

15 paper, we demonstrated that, viewed on an evolutionary timescale, the coexistence of competing species enabled by a compensatory balance between competitive abilities and other life-history traits can be collapsed by evolution of a life-history trait driven by their competitive interaction. 
We focused on the maturation rate as the selection target. The maturation rate is a key life-history trait that influences the life schedule and fecundity of the mature individual (Sterns 1992). Either a higher or a lower maturation rate can have both positive and negative effects on viability. A higher maturation rate allows the pre-reproduction period 5 (juvenile stage) to be shortened, thus enhancing the potential survival probability from juvenile to adult, but it has a negative effect on the potential birth rate owing to the precocity of the adult, and vice versa. Certain combinations of nonidentical maturation rates in two competing species balance their competitive abilities and their potential viability, making their coexistence possible. species leads to differences of population structure (relative numbers of adults and juveniles) and total population density at ecological equilibrium. Such an ecological equilibrium state affects the evolution of the maturation rate. In other words, the population structure and the life-history trait both change the selection pressure on the life-history trait (Nelson et al. 2005).

The trade-off between maturation rate and fecundity determines the evolutionary direction of the maturation rate. The evolution of the maturation rate is the process by which less viable individuals are replaced by more viable individuals. That is, the 
process by which a population consisting of individuals with a fairly low maturation rate is replaced by individuals with a higher maturation rate, or vice versa. The population structures and relative densities of the competing species determined by their competitive abilities and maturation rates at ecological equilibrium affect the speed at 5 which an invading mutant replaces the resident population in each species, and this evolutionary process then determines the new population structure and the relative density of the competing species at a new ecological equilibrium. We thus recognize a complex relation between events on ecological and evolutionary timescales.

Previous studies have concluded that competitive interaction on an ecological timescale results in the evolution of those traits directly related to interference or exploitative competition (MacArthur \& Levins 1967; May \& MacArthur 1972; Roughgarden 1972; Slatkin 1980; Case 1981; Taper \& Case 1985, 1992a, 1992b; Abrams \& Chen 2002b). Our results suggest that competitive interaction on an ecological timescale also leads to the evolution of life-history traits that are not directly related to interference or exploitative competition.

In our analysis, the initial condition of the life-history traits allows stable coexistence at an ecological timescale, but the competing species are not in evolutionary equilibrium. Such an initial situation might be realized by the elimination of some species from a 
community at evolutionary equilibrium, or by the introduction of an invader species into such a community (Bohn et al. 2004; Lambrinos 2004).

In the focal case of our analysis, the superior competitor species is excluded by the inferior competitor species during the evolutionary process if the maturation size 5 (maturation rate) of the superior species is much larger than that of the inferior species. That condition is validated by some empirical supporting evidence (Farji-Brener et al. 2004; Richter-Boix et al. 2004). For instance, smaller ants are often more successful in accessing food baits (Farji-Brener et al. 2004). In addition, small Bufo tadpoles, which are superior exploitative competitors than the larger Pelodytes tadpoles, have a shorter larval period, whereas the larval period of the latter species is longer (Richter-Boix et al. 2004).

In the context of the theory of the coexistence of competing species, at least, our results have important implications. They suggest that competitive coexistence maintained by a compensatory balance between competitive abilities and other 15 life-history traits at ecological equilibrium is fragile when considered in light of the evolutionary process. In addition, they suggest the evolutionarily coexistence requires the trade-off between competitive ability and non evolutionary fragile life-history trait. Kisdi and Liu (in press) conduced a similar mechanism of the evolutionarily 
coexistence of two-predator strategies in a predator-prey system. This resemblance may shadow a general evolutionarily coexistence mechanism.

\section{Acknowledgements}

We would like to thank Akira Sasaki, Midori Tsuda, Kazunori Sato, Yoshinari Tanaka

and Yoh Iwasa for their valuable comments.

\section{References}

Abrams P.A. (2000) Character Shifts of Prey Species That Share Predators. Am. Nat., 156, S45-S61

Abrams P.A. \& Chen X. (2002a) The effect of competition between prey species on the evolution of their vulnerabilities to shared predator. Evolutionary Ecology Research, 4, 897-909

Abrams P.A. \& Chen X. (2002b) The evolution of traits affecting resource acquisition and predator vulnerability: character displacement under real and apparent competition. Am. Nat., 160, 692-704

15 Armstrong R.A. (1979) Prey species replacement along a gradient of nutrient enrichment: graphical considerations. Ecology, 60, 76-84

Bohn T., Sandlund O.T., Amundsen P.-A. \& Primicerio R. (2004) Rapidly changing life history during invasion. Oikos, 106, 138-150 
Case T.J. (1981) Niche packing and coevolution in competition communities. Proc. Nat. Acad. Sci. USA, 78, 5021-5025

Chase J.M. \& Leibold M.A. (2003) Ecological niches. University of Chicago Press, Princeton.

5 Dieckmann U. (1997) Can adaptive dynamics invade? Trends Ecol. Evol., 12, 128-131

Dieckmann U. \& Law R. (1996) The dynamical theory of coevolution: a derivation from stochastic ecological processes. J. Math. Biol, 34, 579-612

Eshel I. (1983) Evolutionary and continuous stability. J. theor. Biol, 185, 333-343

Farji-Brener A.G., Barrantes G. \& Ruggiero A. (2004) Environmental rugosity, body size and access to food: a test of the size-grain hypothesis in tropical litter ants. Oikos, 104, 165-171

Gause G.F. (1934) The struggle for existence. Hafner, New York.

Grover J.P. (1997) Resource competition. Chapman and Hall, London.

Geritz S.A.H., Metz J.A.J., Kisdi E. \& Meszena G. (1997) Dynamics of adaptation and evolutionary branching. Phys. Rev. Lett., 78, 2024-2027

Geritz S.A.H., Kisdi E. \& Meszena G. \& Metz J.A.J. (1998) Evolutionary singular strategies and the adaptive growth and branching of the evolutionary tree. Evol. Ecol, 12, 35-57 
Hutchinson G.E. (1951) Copepodology for the ornithologist. Ecology, 32, 571-577

Hutchinson G.E. (1957) Concluding remarks. Cold Spring Harbour Symposium on Quantitative Biology. 22, 415-427

Hutchinson G.E. (1978) An introduction to population ecology. Yale University Press, New Haven.

Kisdi E. \& Liu S. Evolution of handling time can destroy the coexistence of cycling predators. J. Evol. Biol, in press

Kneitel J.M. \& Chase J.M. (2004) Trade-offs in community ecology: Linking spatial scales and species coexistence. Ecology Letters, 7, 69-80

Lambrinos J.G. (2004) How interactions between ecology and evolution influence contemporary invasion dynamics. Ecology, 85, 2061-2070Levin J.M. \& Rees M. (2002) Coexistence and relative abundance in annual plant assemblages: the roles of competition and colonization. Am. Nat., 160, 452-467

Levine J.M. \& Rees M. (2002) Coexistence and relative abundance in annual plant assemblages: the roles of competition and colonization. Am. Nat., 160, 452-467

Levins R. \& Culver D. (1971) Regional coexistence of species and competition between rare species. Proc. Nat. Acad. Sci. USA, 68, 1246-1248

Lotka A.J. (1925) Elements of Physical Biology. Williams \& Wilkins, Baltimore. 
MacArthur R.H. (1958) Population ecology of some warblers of northeastern coniferous forests. Ecology, 39, 599-618

MacArthur R.H. (1972) Geographical Ecology. Princeton University Press, New Jersey.

MacArthur R.H. \& Levins. R. (1967) The limiting similarity, convergence and divergence of coexisting species. Am. Nat., 101, 377-385

May R.M. \& MacArthur R.H. (1972) Niche overlap as a function of environmental variability. Proc. Nat. Acad. Sci. USA, 69, 1109-1113

Maynard Smith J. (1982) Evolution and the Theory of Games. Cambridge: Cambridge University Press.

Mougi A. \& Nishimura K. (2005) Coexistence of competitive species with a stage-structured life cycle. Ecological Research, 20, 581-589

Nakajima T. \& Kurihara Y. (1994) Evolutionary changes of ecological traits of bacterial populations through predator-mediated competition. Oikos, 71, 24-34

Nee S. \& May R.M. (1992) Dynamics of metapopulations: habitat destruction and competitive coexistence. J. Anim. Ecol., 61, 37-40

Nelson W.A., McCauley E. \& Wrona F.J. (2005) Stage-structured cycles promote genetic diversity in a predator-prey system of Daphnia and algae. Nature, 433, $413-417$ 
Paine R.T. (1966) Food web complexity and species diversity. Am. Nat., 100, 65-75

Pianka E.R. (1976) Competition and niche theory. In: Theoretical Ecology (ed. May RM). Sinauer, Sunderland, MA.

Richter-Boix A., Llorente G.A. \& Montori A. (2004) Responses to competition effects of two anuran tadpoles according to life-history traits. Oikos, 106, 39-50

Roff D.A. (2000) Trade-offs between growth and reproduction: an analysis of the quantitative genetic evidence. J. Evol. Biol, 13, 434-445

Roughgarden J. (1972) Evolution of niche width. Am. Nat., 106, 683-718

Slatkin (1980) Ecological character displacement. Ecology, 61, 163-177

10 Sterns S.C. (1977) The evolution of life-history traits. Annu. Rev. Ecol. Syst., 8, 145-171

Sterns S.C. (1992) The Evolution of Life Histories. Oxford University Press.

Taper M.L. \& Case T.J. (1985) Quantitative genetic models for the coevolution of character displacement. Ecology, 66, 355-371

Taper M.L. \& Case T.J. (1992a) Coevolution among competitors. Oxford surveys in Evolutionary Biology.

Taper M.L. \& Case T.J. (1992b) Models of character displacement and the theoretical robustness of taxon cycles. Evolution, 46, 317-333

Tilman D. (1982) Resource competition and community structure. Princeton University 
Press, Princeton.

Tilman D. \& Pacala S. (1993) The maintenance or species richness in plant communities. University of Chicago Press, Chicago, IL.

Vandermeer J.H. (1972) Niche theory. Annual Review of Ecology and Systematics, 3,

Werner E.E. \& Gilliam J.F. (1984) The ontogenetic niche and species interactions in size-structured populations. Annu. Rev. Ecol. Syst., 15, 393

Wilbur H.M. (1980) Complex life cycles. Annu. Rev. Ecol. Syst., 11, 67-93

Yoshida T., Jones L.E., Ellner S.P., Fussmann G.F. \& Hairston N.G., Jr. (2003) Rapid evolution drives ecological dynamics in a predator-prey system. Nature, 424, $303-306$

Appendix A Change of equilibrium densities with the increasing maturation rate of the inferior competitor We focus only on adult equilibrium densities because these are proportional to the juvenile equilibrium densities $\left(X^{*}=\left(\gamma_{x} / c_{x}\right) x^{*}\right.$ and $\left.Y^{*}=\left(\gamma_{y} / c_{y}\right) y^{*}\right)$. The evolution of a higher maturation rate in the inferior competitor species changes other parameters that relate to its viability, that is, the juvenile period, $L\left(\gamma_{y}\right)=1 / \gamma_{y}$, the potential probability of 
survival to adulthood, $\tilde{M}\left(\gamma_{y}\right)=\gamma_{y} /\left(\gamma_{y}+\phi\right)$, and the intrinsic birth rate, $b\left(\gamma_{y}\right)=b_{y}-\lambda_{y} \gamma_{y}$.

In the competitive system (eqs. (1a-1d)), the nontrivial equilibrium densities are:

$$
\begin{aligned}
& X^{*}=\frac{U}{G} \cdot\left\{-\frac{H_{2}\left(\gamma_{y}\right)+1}{1-H_{1}\left(\gamma_{y}\right)}+\frac{\alpha\left(\gamma_{x} L\left(\gamma_{y}\right)+v_{x}\right)}{\left(v_{x}-v_{x}^{\prime}\right)}\right\}, \\
& Y^{*}=\frac{U}{G} \cdot\left\{-\frac{\left(1+v_{x}\right)}{\left(v_{x}-v_{x}^{\prime}\right)}+\frac{\beta\left(H_{3}\left(\gamma_{y}\right)+1\right)}{1-H_{1}\left(\gamma_{y}\right)}\right\},
\end{aligned}
$$

where $U=\left(v_{x}-v_{x}^{\prime}\right)\left(v_{y}-v_{y}^{\prime}\right), G=\alpha \beta\left(\gamma_{x} / \gamma_{y}+v_{x}\right)\left(\gamma_{y} / \gamma_{x}+v_{y}\right)-\left(1+v_{x}\right)\left(1+v_{y}\right), v_{x}=$ $b\left(\gamma_{x}\right) \gamma_{x}^{2} / c_{x}^{2}, v_{y}=b\left(\gamma_{y}\right) \gamma_{y}^{2} / c_{y}^{2}, v_{x}^{\prime}=\gamma_{x}\left(\gamma_{x}+\phi\right) / c_{x}, v_{y}^{\prime}=\gamma_{y}\left(\gamma_{y}+\phi\right) / c_{y}, H_{1}\left(\gamma_{y}\right)=c_{y} / b\left(\gamma_{y}\right) \tilde{M}\left(\gamma_{y}\right)$, $H_{2}\left(\gamma_{y}\right)=c_{y}^{2} L\left(\gamma_{y}\right)^{2} / b\left(\gamma_{y}\right), H_{3}\left(\gamma_{y}\right)=c_{y}^{2} L\left(\gamma_{y}\right) / \gamma_{x} b\left(\gamma_{y}\right)$, and $\tilde{M}\left(\gamma_{y}\right)=\gamma_{y} /\left(\gamma_{y}+\phi\right)$ (see Mougi and Nishimura 2005). (In the following analysis, $c_{x}=c_{y}=c, b_{x}=b_{y}=b$, and $\lambda_{x}=\lambda_{y}=\lambda$.)

$v_{i}-v_{i}^{\prime}>0(i=x$ or $y)$ is the persistence condition of each species (see Mougi \& Nishimura 2005), which is required for coexistence of competing species. Note that $U$ and $G$ are common terms among the equilibrium values. Thus, we can determine changes in the equilibrium relative densities as the maturation rate of the inferior competitor, $\gamma_{y}$, increases by focusing only on the terms within the braces. In the coexistence region (i.e., in the region where values of $\gamma_{x}$ are large and values of $\gamma_{y}$ are small), if $U / G<0$, then the terms within the braces are negative. Therefore, we examine the change in the absolute values of the terms within the braces with increasing $\gamma_{y}$. Neglecting $U / G$, eqs. (A1) and (A2) become 
$X^{*} \propto\left|A_{X}+P_{X}\right|$,

$Y^{*} \propto\left|A_{Y}+P_{Y}\right|$,

where $A_{X}=-\frac{H_{2}\left(\gamma_{y}\right)+1}{1-H_{1}\left(\gamma_{y}\right)}, P_{X}=\frac{\alpha\left(\gamma_{x} L\left(\gamma_{y}\right)+v_{x}\right)}{\left(v_{x}-v_{x . \text { inf }}^{\prime}\right)}, A_{Y}=-\frac{\left(1+v_{x}\right)}{\left(v_{x}-v_{x . \text { inf }}^{\prime}\right)}$, and $P_{Y}$ $=\frac{\beta\left(H_{3}\left(\gamma_{y}\right)+1\right)}{1-H_{1}\left(\gamma_{y}\right)}$. In (A3) and (A4), the anterior term $\left(A_{X}\right.$ and $\left.A_{Y}\right)$ is negative and the

5 posterior term $\left(P_{X}\right.$ and $\left.P_{Y}\right)$ is positive. We survey the signs of the derivative of each term with respect to $\gamma_{y}$ to evaluate the change in the total absolute magnitude of eqs. (A3) and (A4) with the change in $\gamma_{y}$. It can be easily seen that $\mathrm{d} P_{X} / \mathrm{d} \gamma_{y}<0$ and $\mathrm{d} A_{Y} / \mathrm{d} \gamma_{y}=0$.

If $\mathrm{d}\left(-A_{X}\right) / \mathrm{d} \gamma_{y}<0$ and $\mathrm{d} P_{Y} / \mathrm{d} \gamma_{y}<0$ are satisfied in the focal coexistence region (i.e., relatively large values of $\gamma_{x}$ and relatively small values of $\gamma_{y}$ ), then we can deduce that

$10 X^{*}$ decreases and $Y^{*}$ increases with increasing $\gamma_{y}$. The necessary and sufficient condition to satisfy the conditions $\mathrm{d}\left(-A_{X}\right) / \mathrm{d} \gamma_{y}<0$ and $\mathrm{d} P_{Y} / \mathrm{d} \gamma_{y}<0$ is $\left(1-H_{1}\left(\gamma_{y}\right)\right) H_{i}^{\prime}\left(\gamma_{y}\right)+(1$ $\left.+H_{i}\left(\gamma_{y}\right)\right) H_{1}{ }^{\prime}\left(\gamma_{y}\right)<0,(i=2$ or 3$)$. We can see that for $\gamma_{y}$ such that $\lambda \gamma_{y}{ }^{4}+2 \lambda \phi \gamma_{y}{ }^{3}$ $+(3 c \lambda-b \phi) \gamma_{y}^{2}-2 c(b-c) \gamma_{y}+c^{2} \phi<0$ holds, $\mathrm{d}\left(-A_{X}\right) / \mathrm{d} \gamma_{y}<0$, and for $\gamma_{y}$ such that $\lambda \gamma_{x} \gamma_{y}^{2}$ $+2 \lambda\left(c+\gamma_{x} \phi\right) \gamma_{y}+\left(c^{2}-b\left(c+\gamma_{x} \phi\right)<0\right.$ holds, $\mathrm{d} P_{Y} / \mathrm{d} \gamma_{y}<0$. The numerical investigation tells us that the necessary and sufficient condition of $\gamma_{y}$ such that $\mathrm{d}\left(-A_{X}\right) / \mathrm{d} \gamma_{y}<0$ and $\mathrm{d} P_{X} / \mathrm{d} \gamma_{y}<0$ is satisfied in the coexisting region of the $\gamma_{x}-\gamma_{y}$ plane where $\gamma_{y}$ increases during the evolutionary process. Thus, as the maturation rate of the inferior competitor 
evolves, $X^{*}$ decreases and $Y^{*}$ increases. Although these processes operate irrespective of the inferior competitors's competition coefficient, $\alpha$, the content within the braces of (A1) approaches zero if $\alpha$ is relatively large $(\alpha \lesssim 1)$, but it does not if $\alpha$ is small, because the minimum value of $-A_{X}$ does not approach the value of $P_{X}$ if $\alpha$ is small.

\section{Appendix B Condition of unfeasible evolutionarily stable coexistence}

At the singlular point, $\left(\tilde{\gamma}_{x}, \tilde{\gamma}_{y}\right),\left.\frac{\partial W_{i}\left(\hat{\gamma}_{i}, \tilde{\gamma}_{i}\right)}{\partial \hat{\gamma}_{i}}\right|_{\hat{\gamma}_{i}=\tilde{\gamma}_{i}}=0$ is satisfied for $i=x$ and $y$ simultaneously. Assuming symmetry of all parameters except for competition coefficients, we found that $\left.\frac{\partial W_{x}\left(\hat{\gamma}_{x}, \tilde{\gamma}_{x}\right)}{\partial \hat{\gamma}_{x}}\right|_{\hat{\gamma}_{x}=\tilde{\gamma}_{x}}=\left.\frac{\partial W_{y}\left(\hat{\gamma}_{y}, \tilde{\gamma}_{y}\right)}{\partial \hat{\gamma}_{y}}\right|_{\hat{\gamma}_{y}=\tilde{\gamma}_{y}}=0$ is satisfied if and 10 only if $\tilde{\gamma}_{x}=\tilde{\gamma}_{y}$.

We investigated convergence stability of the singular point. The singular strategy is convergence stable if $\frac{d}{d \gamma_{i}}\left[\left.\frac{\partial W_{i}}{\partial \hat{\gamma}_{i}}\right|_{\hat{\gamma}_{i}=\gamma_{i}}\right]_{\gamma_{i}=\tilde{\gamma}}<0$ (Eshel 1983). This condition is fulfilled if $(2 c+\gamma)(b(\gamma)-c)+b(\gamma)\left\{c+\gamma^{2} \phi / c+\gamma(\gamma+3 \phi)\right\}>0$. This ineq. is always satisfied because that $b(\gamma)>c$ is necessarily satisfied for persisting the population of each species. The evolutionary mechanism of the maturation rates leads maturation rates identical between the competing species, and thus, all parameters except for competition coefficients becomes symmetrical between the species. 
We obtain the ecological equilibrium densities at the evolutional singurality,

$X_{s}^{*}=Q(-1+\alpha)$,

$Y_{s}^{*}=Q(-1+\beta)$,

where $Q=\left\{-\gamma\left(c(\gamma \phi+c(\gamma+2 \phi))+\gamma^{2}(c+\gamma) b^{\prime}(\gamma)\right)\right\} /\left\{(-1+\alpha \beta)\left(c^{2}(2 c+\gamma)-\gamma^{3}(c+\gamma)\right.\right.$

$\left.\left.5 \quad b^{\prime}(\gamma)\right)\right\}$. We find that the evolutionarily coexistence, $X_{s}^{*}>0$ and $Y_{s}^{*}>0$, cannot occur when the type of competition is the dominance competition (we now assume $\alpha<1$ and $\beta>1)$ and all parameters except for competition coefficients are symmetric.

We found that asymmetry in some other life history trait than maturation rates which compensates for the asymmetry of competition is necessary for evolutionarily coexistence. In Fig. 2 (c), we showed an example where we assume the asymmetry of $c$.

Appendix C Evolutionary stability of the singular point where two competitors coexist

We survey whether the singular point where two competitors coexist (the trait set that converges into the coexistence equilibrium point of Fig 2 (c)) is ESS. We can evaluate the evolutionary stability of the singular point by using the following vector criterion (Maynard Smith 1982), 


$$
\left(f_{x}, f_{y}\right)=\left(\left.\frac{\partial^{2} W_{x}\left(\hat{\gamma}_{x}, \tilde{\gamma}_{x}, \tilde{\gamma}_{y}\right)}{\partial \hat{\gamma}_{x}^{2}}\right|_{\hat{\gamma}_{x}=\tilde{\gamma}_{x}},\left.\frac{\partial^{2} W_{y}\left(\hat{\gamma}_{y}, \tilde{\gamma}_{y}, \tilde{\gamma}_{x}\right)}{\partial \hat{\gamma}_{y}{ }^{2}}\right|_{\hat{\gamma}_{y}=\tilde{\gamma}_{y}}\right)
$$

If $(\mathrm{C} 1)$ is a negative vector, then the singular point is evolutionarily stable, and if $(\mathrm{C} 1)$ is a positive vector, then the singular point is evolutionarily unstable (evolutionary branching occurs). We have

$\left.5 \quad \frac{\partial^{2} W_{i}\left(\hat{\gamma}_{i}, \tilde{\gamma}_{i}, \tilde{\gamma}_{j}\right)}{\partial \hat{\gamma}_{i}^{2}}\right|_{\hat{\gamma}_{i}=\tilde{\gamma}_{i}}=-\frac{2 b_{i}\left(X^{*}, Y^{*}\right)\left\{\gamma_{i} \phi_{i}\left(x^{*}, y^{*}\right)+c_{i}\left(\gamma_{i}+3 \phi_{i}\left(x^{*}, y^{*}\right)\right)\right\}}{\left(c_{i}+\gamma_{i}\right)\left(\gamma_{i}+\phi_{i}\left(x^{*}, y^{*}\right)\right)^{2}}$,

where $(i, j)=(x, y)$ or $(i, j)=(y, x)$, and $X^{*}, Y^{*}, x^{*}$, and $y^{*}$ are functions of $\tilde{\gamma}_{x}$ and $\tilde{\gamma}_{y}$. Note that $b_{i}\left(X^{*}, Y^{*}\right)>0, \phi_{i}\left(x^{*}, y^{*}\right)>0$ and the other parameter values are positive. Consequently, (C1) is always a negative vector, and the singular point is thus always ESS. 


\section{Figure Legends}

Figure 1: Ecological equilibrium states in $\gamma_{x}-\gamma_{y}$ space. (a) $\alpha=0.1, \beta=1.1$; (b) $\alpha=0.9$, $\beta=1$.1. In both (a) and (b), $c=1, \phi=0.1, b_{x}=b_{y}=4$, and $\lambda_{x}=\lambda_{y}=1$. In regions $I a$ and $I b$, both species stably coexist. In the shaded areas, the trivial equilibrium state is $X^{*}+x^{*}$ $5>0$ and $Y^{*}+y^{*}<0$, or $X^{*}+x^{*}<0$ and $Y^{*}+y^{*}>0$.

Figure 2: Selection and evolution of $\gamma_{x}$ and $\gamma_{y}$. The parameter values in (a) and (b) are identical to those in Fig. 1 . (c) $\alpha=0.5, \beta=1.1, c_{x}=1.5, c_{y}=1, \phi=0.1, b_{x}=b_{y}=4$, and $\lambda_{x}=\lambda_{y}=1$. (a), (b-1), (b-2), and (c) show the selection vector fields based on the magnitude and sign of the invasion fitness of the maturation rates of each species. (b2)

10 is an enlargement of the indicated portion of (b1). In case (b), simulated evolutionary dynamics for the maturation rates are shown (see (b3)). The simulation starts at the gray circles and terminates at the time when either species becomes extinct (open circles). The initial states of the upper and lower evolutionary trajectory are $\left(\gamma_{x}, \gamma_{y}\right)=(2.4,0.1)$ and $\left(\gamma_{x}, \gamma_{y}\right)=(2.4,0.07)$ in $\operatorname{tr}-1$ and $\operatorname{tr}-2$, respectively. The dashed line on panel (b3) indicates the separatrix, above which evolutionary trajectory leads to extinction of the superior species and below which trajectory leads to extinction of the inferior species. The shaded contours indicate the ratio of the superior competitor's population density to its total abundance $\left(\left(X^{*}+x^{*}\right) /\left(X^{*}+x^{*}+Y^{*}+y^{*}\right)\right)$. The lighter the contour, the higher the 
superior competitor's abundance.

Figure 3: Population densities of the two species in a region of coexistence for given values of $\gamma_{x}$ and $\gamma_{y}$. The parameter values are identical to those of Fig. 1(b), and $\gamma_{x}=2$. (a) The superior competitor's equilibrium densities; (b) the inferior competitor's equilibrium densities. In both graphs, the dotted line, dashed line, and solid line indicate juvenile, adult, and total population equilibrium densities, respectively. 
Figure 1

5

(a)

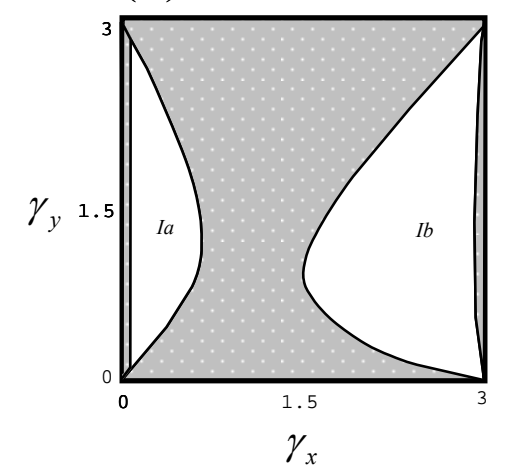

(b)

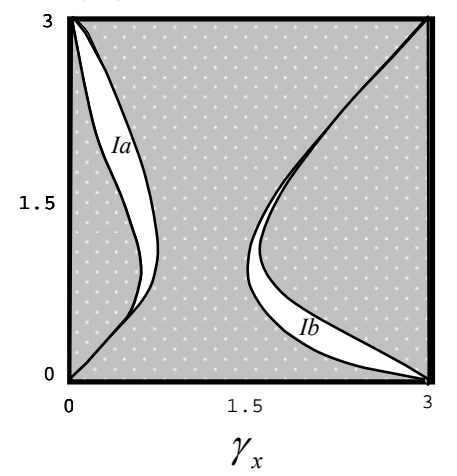


Figure 2

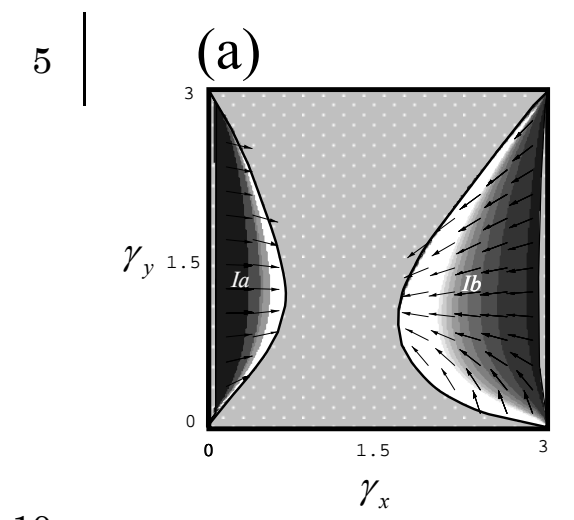

10

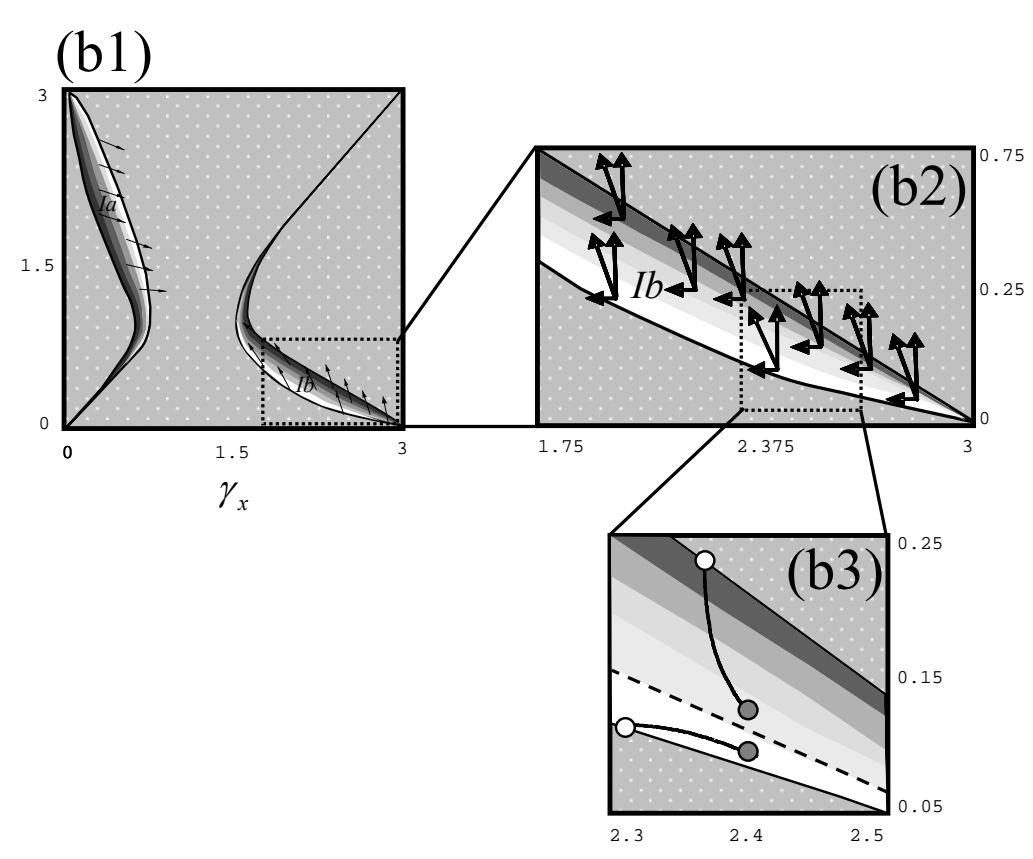

15

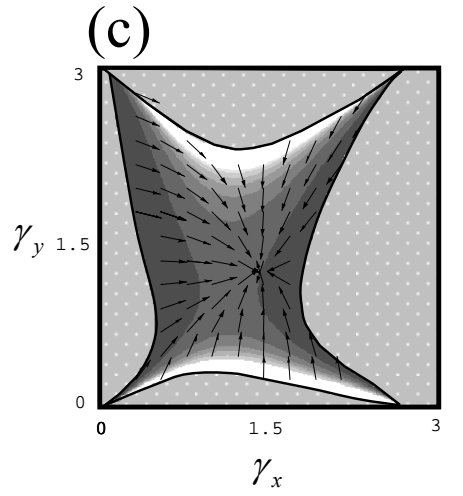


Figure 3
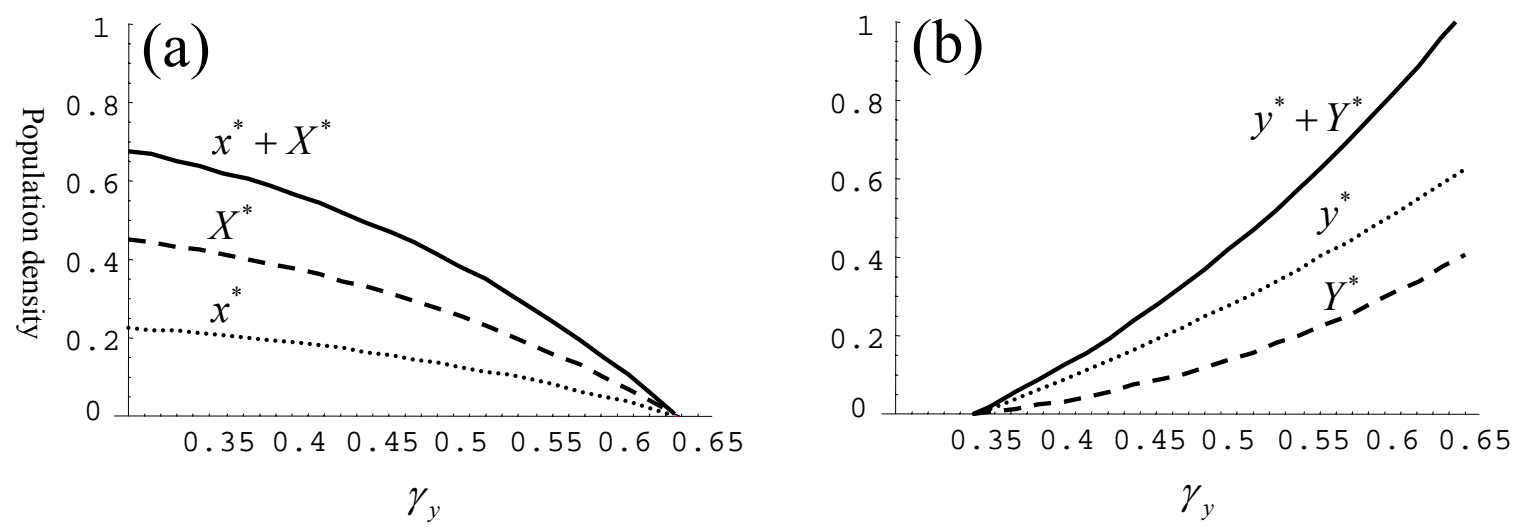